Review Article

\title{
Species Diversity and Floristic Analysis of the Family Poaceae in Libya Depending on the Flora of Libya
}

\author{
Fathi Goma Al-Sghair*, Mohammed Hadi Mahklouf, Ebtisam Ali Abudaya \\ Department of Botany, Faculty of Sciences, University of Tripoli, Tripoli, Libya \\ Email address: \\ fathi_alsghair@yahoo.com (F. G. Al-Sghair).mahklouf64@yahoo.com (M. H. Mahklouf), ebtisamali@yahoo.co.uk (E. A. Abudaya) \\ ${ }^{*}$ Corresponding author
}

\section{To cite this article:}

Fathi Goma Al-Sghair, Mohammed Hadi Mahklouf, Ebtisam Ali Abudaya. Species Diversity and Floristic Analysis of the Family Poaceae in Libya Depending on the Flora of Libya. Advances in Bioscience and Bioengineering. Vol. 7, No. 2, 2019, pp. 13-21.

doi: 10.11648/j.abb.20190702.11

Received: June 8, 2019; Accepted: July 9, 2019; Published: July 23, 2019

\begin{abstract}
The goal of this research was to investigate the species diversity and floristic analysis of the family Poaceae in Libya depending on the data provided from the Flora of Libya series. 3 species (Bromus unioloides (Willd.) H. B. K., Eriochloa fatmensis (Hochst. \& Steud.) Clayton and Chloris gayana Kunth.) were added as a new record to the family Poaceae in Libya. Results revealed that the family Poaceae in Libya is composed of 229 species belonging to 92 genera. Simpson's Diversity index showed that the Family Poaceae has high diversity. The largest genera in the Family Poaceae in the flora of Libya are Stipagrostis and Bromus, which include 13 species. There are (13 species) of Stipagrostis in Libya (26\%) of 50 species in the world. The life forms and chorological spectra of plant species were determined. There are no trees and shrubs species in our data, this due to the difficulties for the species to grow in dry habitat. It appears that annual and perennial life forms are the preferable strategy in the temperate deserts of Libya. Therophytes showed the maximum number of species $(62.2 \%)$, followed by Hemicryptophytes (19.65\%), Geophytes (13.5\%) and Therophytes - Hemicryptophytes $(2.62 \%)$. The results obtained from the geographical distribution of the species showed that the highest percentage is (31.88\%) for the Mediterranean region, followed by (14.85\%) Mediterranean / Irano-Turanian regions. Distribution of species clearly shows that the majority of species of the family Poaceae are located within the Mediterranean region.
\end{abstract}

Keywords: Flora of Libya, Poaceae, Species Diversity, Floristic Analysis, Life Forms-Chorotype

\section{Introduction}

The grasses (Poaceae) are certainly the most important plant family for food production [1] to mankind agriculturally, economically and ecologically. The Family Poaceae contains approximately 11000 species [2] distributed among about 750-770 genera [3-4] worldwide and is the fifth largest flowering plant family $[5,6,7]$. It covers about $40 \%$ of the Earth's surface [8]. Poaceae members are annual or perennial herbs with fibrous roots and often rhizomes [9]. It provides the major cereal crops and most of the grazing for wild and domestic herbivores such as, wheat (Triticum aestivum), rice (Oryza sativa), maize (Zea mays), sugar cane (Saccharum officinarum), [10], and barley (Hordeum vulgare) [11], which is the fourth most important cereal crop in terms of planting area and is mainly used in brewing industries and as forage [12]. Grasses have adapted to conditions in rain forests, dry deserts, and cold mountain steppes, and are now the most widespread plant type [9], which make up $20 \%$ of the world's vegetation coverage and are composed of Poaceae members [13-14].

Libya is a country in the North African region. It lies along the southern coast of the Mediterranean, approximately between latitude $18^{\circ}$ and $33^{\circ}$ North and $9^{\circ}$ and $25^{\circ}$ East (Figure 1) and occupies an area of about 1, 759, 540 square kilometres [15], more than $90 \%$ of which is deserted, except the coastal strip and Al Jabal El-Akhdar and Jabal Nafousa regions [16]. According to Boulos [17], the coastal belt which extends from the Tunisian to the Egyptian borders is about 5.2\% of the whole country. This area is quite fertile and receives an 
adequate amount of rainfall in winter, particularly in the east and west, thus a great part of this belt exhibits the typical Mediterranean flora. In Libya, four Biogeographical regions are recognized, which are Sudanian region, Saharo- Arabian region, the Mediterranean region and Mauritanian steppe of Irano-Turanian region [18]. The climate is typical of the Mediterranean, characterized by the cool, rainy winter season and a hot dry summer. The climate over most of the country is that of the hot, arid Sahara, but it is moderated along the coastal littoral by the Mediterranean Sea [19]. This study is based on the analysis of the family Poaceae (Graminae) by [20] in Flora of Libya series.



Figure 1. Map of Libya. (Via https://www.google.co.uk/map of Libya)

\section{Species Diversity}

Species diversity is one of the most important indices which are used for the evaluation of ecosystems at different scales [21]. Biodiversity measurement typically focuses on the species level and local diversity can be studied with various indices [22]; such as species richness or Simpson's index which are commonly used to evaluate different trends in plant diversity. Simpson's index of Diversity values range between 0 and 1 ; when the value closer to 1 it is more diverse and when it closer to 0 it is less diverse [23-24].

In this study, Simpson's diversity index calculates a diversity score for the family Poaceae; it is based on both the number of different species of each genus and the number of individuals present for each of those species (Table 1). The formula for calculating Simpson's index is:

$$
\mathrm{D}=1-\frac{\sum \mathrm{ni}(\mathrm{ni}-1)}{\mathrm{N}(\mathrm{N}-1)}
$$

Where $\mathrm{N}=$ the total number of all species in the family Poaceae.

$\mathrm{ni}=$ the numbers of species of each genus.

$\sum \mathrm{ni}(\mathrm{ni}-1)=(156 \times 2)+(56 \times 3)+42+(30 \times 6)+20+(12 \times 4)$

$+(6 \times 13)+(2 \times 14)+(48 \times 0)$

$=312+168+42+180+20+48+78+28$

$=876$

$\mathrm{N}(\mathrm{N}-1)=229(229-1)=52212$

Simpson's Diversity Index $(D)=1-(876 / 52212)=1-0.02=$ 0.98
The value of (D) ranges between 0 and 1 . With this index, 1 represents very high diversity, and 0 no diversity. The Poaceae is highly diverse depending on the (D) value obtained from calculating Simpson's diversity index.

Table 1. Shows the number of species depending on the genus in the Family Poaceae.

\begin{tabular}{|c|c|c|c|}
\hline Genus & number of species $\left(n_{i}\right)$ & $\left(n_{i}-1\right)$ & $\mathrm{n}_{\mathrm{i}}\left(\mathrm{n}_{\mathrm{i}}-\mathbf{1}\right)$ \\
\hline Stipagrostis & 13 & 12 & 156 \\
\hline Bromus & 13 & 12 & 156 \\
\hline Avena & 8 & 7 & 56 \\
\hline Poa & 8 & 7 & 56 \\
\hline Vulpia & 8 & 7 & 56 \\
\hline Phalaris & 7 & 6 & 42 \\
\hline Aegilops & 6 & 5 & 30 \\
\hline Eragrostis & 6 & 5 & 30 \\
\hline Hordeum & 6 & 5 & 30 \\
\hline Setaria & 6 & 5 & 30 \\
\hline Stipa & 6 & 5 & 30 \\
\hline Triticum & 6 & 5 & 30 \\
\hline Lophochloa & 5 & 4 & 20 \\
\hline Aristida & 4 & 3 & 12 \\
\hline Lolium & 4 & 3 & 12 \\
\hline Pennisetum & 4 & 3 & 12 \\
\hline Trisetaria & 4 & 3 & 12 \\
\hline Catapodium & 3 & 2 & 6 \\
\hline Cutandia & 3 & 2 & 6 \\
\hline Cynosurus & 3 & 2 & 6 \\
\hline Desmazeria & 3 & 2 & 6 \\
\hline Eleusine & 3 & 2 & 6 \\
\hline Elytrigia & 3 & 2 & 6 \\
\hline Panicum & 3 & 2 & 6 \\
\hline Parapholis & 3 & 2 & 6 \\
\hline Piptatherum & 3 & 2 & 6 \\
\hline Polypogon & 3 & 2 & 6 \\
\hline Saccharum & 3 & 2 & 6 \\
\hline Sorghum & 3 & 2 & 6 \\
\hline Sporobolus & 3 & 2 & 6 \\
\hline Aeluropus & 2 & 1 & 2 \\
\hline Aira & 2 & 1 & 2 \\
\hline Alopecurus & 2 & 1 & 2 \\
\hline Ammochloa & 2 & 1 & 2 \\
\hline Asthenatherum & 2 & 1 & 2 \\
\hline Briza & 2 & 1 & 2 \\
\hline Cenchrus & 2 & 1 & 2 \\
\hline Chloris & 2 & 1 & 2 \\
\hline Dichanthium & 2 & 1 & 2 \\
\hline Digitaria & 2 & 1 & 2 \\
\hline Gastridium & 2 & 1 & 2 \\
\hline Schismus & 2 & 1 & 2 \\
\hline Secale & 2 & 1 & 2 \\
\hline Sphenopus & 2 & 1 & 2 \\
\hline Other 48 genus & 1 & 0 & 0 \\
\hline
\end{tabular}

\section{Floristic}

This paper provides an overview of the Family Poaceae depending on the analysis of the flora of Libya, with life form patterns, distribution of species and chorotype.

According to [25], [26], there are 2088 species belonging to 844 genera and 145 families in the flora of Libya as angiosperms. The second dominant family in the flora of Libya is Poaceae with 226 species of 91 genera [20] (Appendix). In addition to Bromus unioloides (Willd.) H. B. K. 
recorded by [27], Eriochloa fatmensis (Hochst. \& Steud.) Clayton. recorded by [28] and Chloris gayana Kunth. recorded by [29], the Family Poaceae in Libya became 229 species belonging to 92 genera. The largest genera in the Family Poaceae in the flora of Libya are Stipagrostis and Bromus, which include 13 species, followed by Vulpia and Poa with 8 species each, Phalaris (7 species), Aegilops, Eragrostis, Hordeum, Setaria, Stipa and Triticum (6 species each), Lophochloa (5 species), Aristida, Lolium, Pennisetum and Trisetaria with (4 species each). There are (13 species) of Stipagrostis in Libya (26\%) of 50 species in the world [15].

\section{Life Forms}

According to Raunkiaer's method [30] which was modified by [31], a high proportion of herbs (annuals then perennials). There are no woody (trees and shrubs) species in our data (Table 2). This can be referred to the difficulties for the species to grow in dry habitat.

Figure 2 shows that the highest life form recorded was for the Therophytes which constituted 147 species representing $(62.2 \%)$ of the total species followed by the Hemicryptophytes with 45 species representing (19.65\%), Geophytes 31 species (13.5\%) and Therophytes Hemicryptophytes 6 species $(2.62 \%)$. Therophytes and Hemicryptophytes are the most frequent life forms which may indicate typical desert spectrum vegetation.

Table 2. Life forms of species.

\begin{tabular}{lll}
\hline Life forms & No. of species & \% of total species \\
\hline Therophytes (T) & 147 & 62.2 \\
Hemicryptophytes (He) & 45 & 19.65 \\
$\begin{array}{l}\text { Therophytes / Hemicryptophytes } \\
\text { (T/He) }\end{array}$ & 6 & 2.62 \\
Geophytes (Ge) & 31 & 13.50 \\
\hline
\end{tabular}

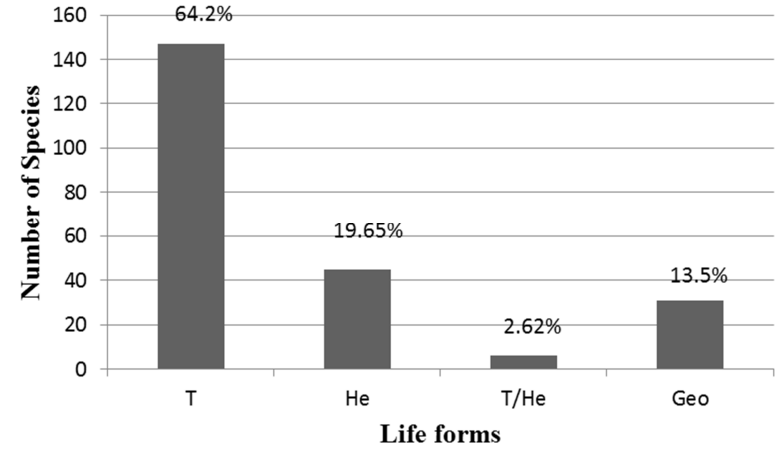

Figure 2. Shows the number of species and percentage of Life forms in the Family Poaceae.

\section{Geographical Elements of Species Level (Chorotype)}

The results of the geographical distribution of the species showed that 73 species $(31.88 \%)$ are dominated in the Mediterranean region (Figure 3). A ratio of $14.85 \%$ (34 species out of the total) belongs to Mediterranean /Irano-Turanian regions, $8.7 \%$ (20 species) belong to tropical / sub-tropical region, $8.3 \%$ (19 species) belongs to Euro Siberian / Mediterranean / Irano -Turanian regions, 14 species $(6.11 \%)$ belong to Mediterranean / Saharo-Arabian regions, $4.8 \%$ (11 species) belong to Saharo-Arabian region, 11 species with a ratio of $4.8 \%$ belong to Cosmopolitan, $2.62 \%$ (6 species) belong to Euro - Siberian / Mediterranean regions. Figure 4 shows species distribution of Poaceae members depending on coordinates have been given by the flora of Libya.

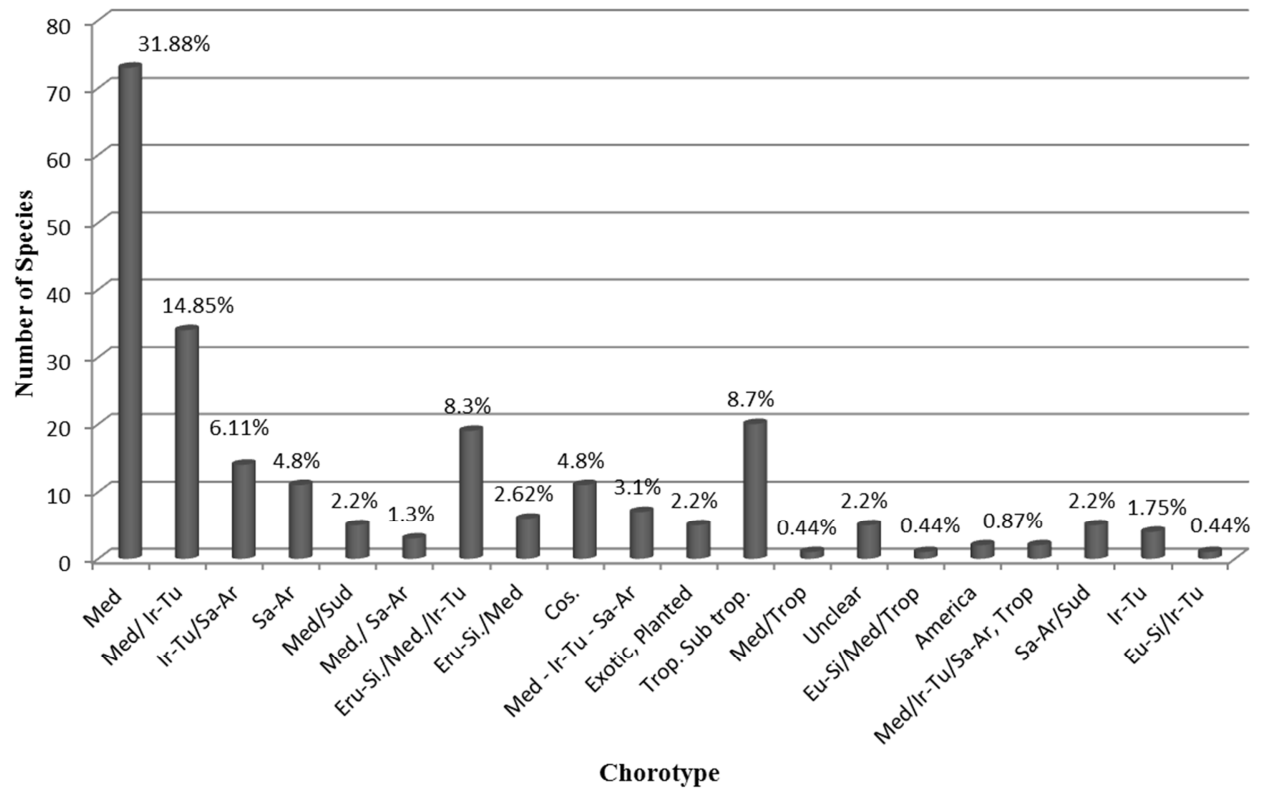

Abbreviations: $\mathrm{Med}=$ Mediterranean, $\mathrm{SaAr}=$ Saharo-Arabain, $\mathrm{IrTu}=$ Irano-Turanian, EuSi $=$ European Siberia, Cos $=$ Cosmopolitan.

Figure 3. Geographical distribution of species showing number \& percentage of species in each Chorotype in Family Poaceae. 


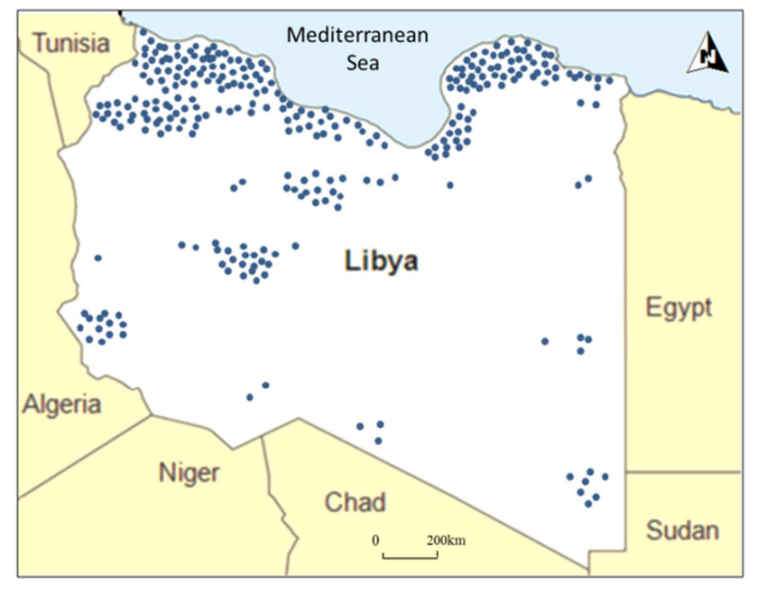

Figure 4. Distribution of Poaceae species depending on the coordinate's flora of Libya.

\section{Discussion}

The results obtained from the calculation of Simpson's index showed that the value of the index is high, the family Poaceae is highly diverse due to the fact that about $52 \%$ of the genera of the Family Poaceae have only one species. The Chorotype of plant species in a region reflects the influence of the different vegetation areas [32]. Our finding showed that chorological characteristic of the Poaceae species showed that Mediterranean region elements recorded the highest percentage $(31.88 \%)$ followed by Mediterranean /Irano-Turanian regions elements (14.85\%). The life forms of plants indicate their taxonomy features and reflect their adaptation with the environmental conditions. According to Raunkiaer's method, the plant life form classes along Family Poaceae indicated the clear dominance of Therophytes $(62.2 \%)$ followed by the Hemicryptophytes (19.65\%). The structure of plant life forms shows their compatibility with habitat conditions for the use of environmental resources in the habitat [33]. The dominance of Therophytes is due to the long dry periods during the year in Libya [15]. It appears that annual and perennial life forms are the preferable strategy in the temperate deserts of Libya. Distribution of species clearly shows that the majority of species of the Family Poaceae are located within the Mediterranean region.

\section{Conclusion}

This study set out to present the first species diversity and floristic study of the family Poaceae in Libya. Simpson's Diversity index showed that it has high diversity; there are 13 species of Stipagrostis in Libya (26\%) out of 50 species in the world. Due to the species' difficulty in growing in dry habitats, there are no trees and shrubs species in our data.

It appears that annual and perennial life forms are the preferable strategy in the temperate deserts of Libya. Therophytes showed the maximum number of species $(62.2 \%)$. Results revealed that the distribution of species clearly shows that the majority of species of the family Poaceae are located within the Mediterranean region.

\section{Appendix}

List of species, Chorotype and Life From based on Sherif and Siddiqi (1988)

\section{Species}

Abdropogon distachyos $\mathrm{L}$.

Aegilops geniculata Roth.

Aegilops kotschyi Boiss.

Aegilops neglecta Reg. ex Bertol.

Aegilops peregrina (Hack.) Maire et Weiller.

Aegilops triuncialis L.

Aegilops ventricosa Tausch.

Aeluropus lagopoides (L.) Trin. Ex Thw.

Aeluropus littoralis (Gouan) Parl.

Aira cupaniana Guss.

Aira tenorii Guss.

Alopecurus mysuroides Huds.

Alopecurus urticulatus Banks et Sol.

Ammochloa palaestina Boiss.

Ammochloa pungens (Schreb.) Boiss.

Ammophila australis (Mabille) Porta et Rigo.

Ampelodesmos mauritanica (Poiret) Th. Dur. \& Schinz.

Antinoria insularis Parl.

Aristida adscensionis L.

Aristida funiculata Trin. et Rupr.

Aristida meccana Hochst.

Aristida mutabilis Trin. Et Rupr.

$\begin{array}{ll}\text { Chorotype } & \text { Life form } \\ \text { Plurireginalbor-trop } & \mathrm{He} \\ \text { Med } & \mathrm{T} \\ \text { Ir-Tu/Sa-Ar } & \mathrm{T} \\ \text { Med } & \mathrm{T} \\ \text { Med } & \mathrm{T} \\ \text { Med/Ir-Tu } & \mathrm{T} \\ \text { Med } & \mathrm{T} \\ \text { Ir-Tu/Sa-Ar } & \mathrm{He} \\ \text { Med/Ir-Tu } & \mathrm{Geo} \\ \text { Med } & \mathrm{T} \\ \text { Med } & \mathrm{T} \\ \text { Eru-Si./Med./Ir-Tu } & \mathrm{T} \\ \text { Med } & \mathrm{T} \\ \text { Med - Ir-Tu - Sa-Ar } & \mathrm{T} \\ \text { Med } & \mathrm{T} \\ \text { Med } & \text { Geo } \\ \text { Med } & \text { Geo } \\ \text { Med } & \mathrm{T} \\ \text { Med - Ir-Tu - Sa-Ar } & \mathrm{T} / \text { hemicry } \\ \text { Ir-Tu/Sa-Ar } & \mathrm{T} \\ \text { Sa-Ar } & \mathrm{T} \\ \text { Ir-Tu/Sa-Ar } & \mathrm{T}\end{array}$




\section{Species}

Arundo donax L.

Asthenatherum forskalii (Vahl.) Nevski.

Asthenatherum fragile (Guinet. et Souvage) Monod.

Avellinia michelii (Savi) Parl.

Avena barbata Pott ex Link.

Avena eriantha Durieu.

Avena fatua $\mathrm{L}$.

Avena longiglumis Durieu.

Avena sativa $\mathrm{L}$.

Avena sterilis $\mathrm{L}$.

Avena ventricosa Balansa ex Cosson.

Avenula bromoides (Gouan) H. Scholz.

Brachypodium retusum (Pers.) P. Beauv.

Briza maxima $\mathrm{L}$.

Briza minor L.

Bromus alopecuros Poir.

Bromus caroli-henrici Greuter.

Bromus chrysopogon Viviani.

Bromus diandrus Roth.

Bromus fasciculatus C. Presl.

Bromus intermedius Guss.

Bromus lanceolatus Roth.

Bromus madritensis L.

Bromus molliformis Lloyd.

Bromus rigidus Roth.

Bromus rubens $\mathrm{L}$.

Bromus scoparius L.

Bromus unioloides (Willd.) H. B. K.

Castellia tuberculosa (Moris) Bor in Ind.

Catabrosa aquatica (L.) P. Beauv.

Catapodium hemipoa (Delile ex Spreng.) Lainz.

Catapodium marinum (L.) C. E. Hub.

Catapodium rigidum (L.) C. E. Hub.

Cenchrus ciliaris L.

Cenchrus incertus M. A. Curtis.

Chloris gayana Kunth

Chloris virgata Swartz.

Corynephorus divaricatus (Pourr.) Breistr.

Crithopsis delileana (Schultes) Rozhev.

Crypsis schoenoides (L.) Lam.

Ctenopsis pectinella (Del.) De Not.

Cutandia dichotoma (Forsk.) Trabut.

Cutandia maritima (L.) Barbey.

Cutandia memphitica (Sreng.) Richter.

Cymbopogon schoenanthus (L.) Spreng.

Cynodon dactylon (L.) Pers.

Cynosurus coloratus Lehm. Ex Steud.

Cynosurus elegans Desf.

Cynosurus junceus Murb.

Dactylis glomerata L.

Dactyloctenium aegyptium (L.) P. Beauv.

Desmazeria lorentii $\mathrm{H}$. Scholz.

Desmazeria philistaea (Boiss.) H. Scholz.

Desmazeria sicula (Jacq.) Dumort.

Desmostachya bipinnata (L.) Stapf.

Dichanthium annulatum (Forsk.) Stapf.

\begin{tabular}{|c|c|}
\hline Chorotype & Life form \\
\hline Med/ Ir-Tu & Geo \\
\hline $\mathrm{Ir}-\mathrm{Tu} / \mathrm{Sa}-\mathrm{Ar}$ & $\mathrm{He}$ \\
\hline Med/Sud & $\mathrm{He}$ \\
\hline Med & $\mathrm{T}$ \\
\hline Med & $\mathrm{T}$ \\
\hline Eru-Si./Med./Ir-Tu & $\mathrm{T}$ \\
\hline Unclear & $\mathrm{T}$ \\
\hline Med./ Sa-Ar & $\mathrm{T}$ \\
\hline Exotic, Planted, Escaped from cultivation & $\mathrm{T}$ \\
\hline Med./ Ir-Tu. & $\mathrm{T}$ \\
\hline $\mathrm{Med} / \mathrm{Ir}-\mathrm{Tu}$ & $\mathrm{T}$ \\
\hline Med & $\mathrm{He}$ \\
\hline Med & Geo \\
\hline Med & $\mathrm{T}$ \\
\hline Eu-Si/Med/Ir-Tu & $\mathrm{T}$ \\
\hline Med & $\mathrm{T}$ \\
\hline Med & $\mathrm{T}$ \\
\hline $\mathrm{Med} / \mathrm{Ir}-\mathrm{Tu}$ & $\mathrm{T}$ \\
\hline Med & $\mathrm{T}$ \\
\hline Med & $\mathrm{T}$ \\
\hline $\mathrm{Med} / \mathrm{Ir}-\mathrm{Tu}$ & $\mathrm{T}$ \\
\hline Med./ Ir-Tu. & $\mathrm{T}$ \\
\hline Med./ Ir-Tu. & $\mathrm{T}$ \\
\hline Eru-Si./Med & $\mathrm{T}$ \\
\hline Med & $\mathrm{T}$ \\
\hline $\mathrm{Med} / \mathrm{Ir}-\mathrm{Tu} / \mathrm{Sa}-\mathrm{Ar}$ & $\mathrm{T}$ \\
\hline $\mathrm{Med} / \mathrm{Ir}-\mathrm{Tu}$ & $\mathrm{T}$ \\
\hline Eu-Si- Med - Ir-Tu & $\mathrm{T}$ \\
\hline Unclear & $\mathrm{T}$ \\
\hline Eu-Si/Med/Ir-Tu & Geo \\
\hline Med & $\mathrm{T}$ \\
\hline Med & $\mathrm{T}$ \\
\hline Med & $\mathrm{T}$ \\
\hline $\mathrm{Sa}-\mathrm{Ar}$ & $\mathrm{He}$ \\
\hline N. and C. America & $\mathrm{T}$ \\
\hline Trop. Africa & $\mathrm{He}$ \\
\hline Subtropical-Tropical & $\mathrm{T}$ \\
\hline Med & $\mathrm{T}$ \\
\hline Med./Ir-Tu & $\mathrm{T}$ \\
\hline $\mathrm{Eu}-\mathrm{Si} / \mathrm{Med} / \mathrm{Ir}-\mathrm{Tu}$ & $\mathrm{T}$ \\
\hline $\mathrm{Sa}-\mathrm{Ar}$ & $\mathrm{T}$ \\
\hline $\mathrm{Ir}-\mathrm{Tu} / \mathrm{Sa}-\mathrm{Ar}$ & $\mathrm{T}$ \\
\hline Med & $\mathrm{T}$ \\
\hline $\mathrm{Ir}-\mathrm{Tu} / \mathrm{Sa}-\mathrm{Ar}$ & $\mathrm{T}$ \\
\hline $\mathrm{Sa}-\mathrm{Ar}$ & $\mathrm{He}$ \\
\hline Plurireginalbor-trop & Geo \\
\hline Med & $\mathrm{T}$ \\
\hline Med./ Ir-Tu. & $\mathrm{T}$ \\
\hline Med & $\mathrm{He}$ \\
\hline Med & Geo \\
\hline Tropical & $\mathrm{T}$ \\
\hline Med & $\mathrm{T}$ \\
\hline Med & $\mathrm{T}$ \\
\hline Med & $\mathrm{T}$ \\
\hline Tropical & Geo \\
\hline Subtropical-Tropical & $\mathrm{He}$ \\
\hline
\end{tabular}




\section{Species}

Dichanthium foveolatum (Del.) Roberty.

Digitaria bicornis (Lam.) Roem. et Schult.

Digitaria sanguinalis (L.) Scop.

Dinebra retroflexa (Vahl) Panz.

Echinaria capitata (L.) Desf.

Echinochloa colona (L.) Link.

Eleusine compressa (Forsk.) Aschers. \& Schw. ex

Eleusine coracana (L.) Gaertn.

Eleusine indica (L.) Gaertn.

Elytrigia juncea (L.) Nevski.

Elytrigia littoralis (Host) Hyl.

Elytrigia repens (L.) Desv. Ex Nevski.

Enneapogon desvauxii P. Beauv.

Eragrostis aegyptiaca (Willd.) Link.

Eragrostis barrelieri Dav.

Eragrostis cilianensis (All.) Vign.-Lutati malpighia

Eragrostis ciliaris (L.) R. Br.

Eragrostis pilosa (L.) P. Beauv.

Eragrostis tef (Zucc.) Trotter.

Eriochloa fatmensis (Hochst. \& Steud.) Clayton

Festuca arundinacea Schreb.

Gastridium scabrum C. Presl.

Gastridium ventricosum (Gouan) Schinz et Thell.

Gaudinia fragilis (L.) P. Beauv.

Hainardia cylindrica (Willd.) Greuter.

Hordeum bulbosum $\mathrm{L}$.

Hordeum distichon $\mathrm{L}$.

Hordeum geniculatum All.

Hordeum marinum Huds.

Hordeum spontaneum C. Koch.

Hordeum vulgare $\mathrm{L}$.

Hyparrhenia hirta (L.) Stapf.

Imperata cylindrica (Linn.) Raeuschel.

Lagurus ovatus $\mathrm{L}$.

Lamarckia aurea (L.) Moench.

Lasiurus hirsutus (Forssk.) Boiss.

Leersia hexandra Swartz.

Libyella cyrenaica (Durand \& Barratte) Pamp.

Lolium loliaceum (Bory \& Chaub) Hand Mazz.

Lolium multiflorum Lam.

Lolium perenne $\mathrm{L}$.

Lolium rigidum Gaud.

Lophochloa cristata (L.) Hyl.

Lophochloa pubescens (Lam.) H. Scholz.

Lophochloa pumila (Desf.) Bor.

Lophochloa rohlfsii (Ascherson) H. Scholz.

Lophochloa salzmannii (Boiss.) H. Scholz.

Lygeum spartum Loefl. ex Linn.

Melica minuta L.

Micropyrum tenellum (L.) Link.

Milium vernale $\mathrm{M}$. Beib.

Oryza sativa L.

Panicum miliaceum $\mathrm{L}$.

Panicum repens $\mathrm{L}$.

Panicum turgidum Forsk.

Parapholis incurva (L.) C. E. Hub.

\section{Chorotype}

Sa-Ar/Sud

Tropical

Plurireginalbor-trop

Tropical

Med

Subtropical-Tropical

$\mathrm{Med} / \mathrm{Ir}-\mathrm{Tu}$

Tropical

Subtropical-Tropical

Med

Eur-Si/Med

Eur-Si/Med/Ir-Tu

$\mathrm{Ir}-\mathrm{Tu} / \mathrm{Sa}-\mathrm{Ar}$

$\mathrm{Med} / \mathrm{Sud}$

$\mathrm{Med} / \mathrm{Sa}-\mathrm{Ar}$

Plurireginalbor-trop

Med/Sud

Borealo-Trop

Unclear

Sa-Ar

Eu-Si/Med/Ir-Tu

Med

Med

Med

Med

$\mathrm{Med} / \mathrm{Ir}-\mathrm{Tu}$

Exotic, Planted, Escaped from cultivation

$\mathrm{Med} / \mathrm{Ir}-\mathrm{Tu}$

$\mathrm{Med} / \mathrm{Ir}-\mathrm{Tu}$

Med./ Ir-Tu.

Exotic, Planted, Escaped from cultivation

$\mathrm{Med} / \mathrm{Ir}-\mathrm{Tu} / \mathrm{Sa}-\mathrm{Ar}$

Med/Ir-Tu/Sa-Ar, Trop

Med

Med/Ir-Tu

$\mathrm{Sa}-\mathrm{Ar} / \mathrm{Sud}$

M./Ir-Tu/Sa-ar/Trop

Med

Med

Med

$\mathrm{Eu}-\mathrm{Si} / \mathrm{Med} / \mathrm{Ir}-\mathrm{Tu}$

Med./ Ir-Tu.

$\mathrm{Med} / \mathrm{Ir}-\mathrm{Tu}$

Med

$\mathrm{Ir}-\mathrm{Tu} / \mathrm{Sa}-\mathrm{Ar}$

$\mathrm{Sa}-\mathrm{Ar}$

Med

Med

Med

Eur-Si/Med

Med

Ir-Tu

Eu-Si/Med/Ir-Tu

Subtropical-Tropical

$\mathrm{Sa}-\mathrm{Ar} / \mathrm{Sud}$

$\mathrm{Med} / \mathrm{Ir}-\mathrm{Tu}$
Life form

Geo

$\mathrm{T}$

$\mathrm{T}$

$\mathrm{T}$

$\mathrm{T}$

$\mathrm{T}$

$\mathrm{He}$

$\mathrm{T}$

T

Geo

Geo

Geo

$\mathrm{He}$

$\mathrm{T}$

$\mathrm{T}$

$\mathrm{T}$

$\mathrm{T}$

$\mathrm{T}$

$\mathrm{T}$

$\mathrm{He}$

Geo

$\mathrm{T}$

$\mathrm{T}$

$\mathrm{T}$

$\mathrm{T}$

Geo

$\mathrm{T}$

$\mathrm{T}$

$\mathrm{T}$

$\mathrm{T}$

$\mathrm{T}$

$\mathrm{He}$

Geo

$\mathrm{T}$

$\mathrm{T}$

$\mathrm{He}$

$\mathrm{He}$

$\mathrm{T}$

$\mathrm{T}$

$\mathrm{T} / \mathrm{He}$

$\mathrm{He}$

$\mathrm{T}$

$\mathrm{T}$

$\mathrm{T}$

$\mathrm{T}$

$\mathrm{T}$

$\mathrm{T}$

Geo

$\mathrm{He}$

$\mathrm{T}$

$\mathrm{T}$

$\mathrm{T}$

$\mathrm{T}$

Geo

Geo

$\mathrm{T}$ 


\section{Species}

Parapholis marginata Runemark.

Parapholis strigosa (Dumort.) C. E. Hub.

Paspalidium geminatum (Forsk.) Stapf.

Paspalum paspalodes (Michx.) Scribn.

Pennisetum americanum (L.) Schumann.

Pennisetum divisum (Forsk. ex Gmel.) Henr.

Pennisetum elatum Hochst. ex Steud.

Pennisetum setaceum (Forssk.) Chiov.

Phalaris aquatica L.

Phalaris brachystachys Link.

Phalaris canariensis L.

Phalaris coerulescens Desf.

Phalaris minor Retz.

Phalaris paradoxa $\mathrm{L}$.

Phalaris truncata Guss.

Phleum subulatum (Savi) Aschers. et Graebn.

Phragmites australis (Cav.) Trin. ex Steud.

Piptatherum coerulescens (Desf.) P. Beauv.

Piptatherum holciforme (Bieb.) Roem. Et Schult.

Piptatherum miliaceum (L.) Cosson.

Poa annua L.

Poa bulbosa L.

Poa infirma Kunth.

Poa pentapolitana $\mathrm{H}$. Scholz.

Poa pratensis L.

Poa sinaica Steud.

Poa trivialis L.

Poa vaginata Pamp. In Arch. Bot.

Polypogon maritimus Willd.

Polypogon monspeliensis (L.) Desf.

Polypogon semiverticillatus (Forsk.) Hyl.

Psilurus incurvus (Gouan) Shinz et Thell.

Saccharum officinarum L.

Saccharum ravennae (L.) Murr.

Saccharum spontaneum L.

Schismus arabicus Nees.

Schismus barbatus (L.) Thell.

Secale cereale L.

Secale montanum Guss.

Setaria adhaerens (Forsk.) Chiov.

Setaria glauca (L.) P. Beauv.

Setaria italica (L.) P. Beauv.

Setaria verticillata (L.) Beauv.

Setaria verticillata $\mathbf{x}$ viridis Lloyd.

Setaria viridis (L.) P. Beauv.

Sorghum bicolor (L.) Moench.

Sorghum halepense (L.) Pers.

Sorghum sudanense (Piper) Stapf.

Sphenopus divaricatus (Gouan) Reichenb.

Sphenopus ehrenbergii Hausskn.

Sporobolus helvolus (Trin.) Th.

Sporobolus spicatus (Vahl) Kunth.

Sporobolus virginicus (L.) Kunth.

Stipa barbata Desf.

Stipa capensis Thumb.

Stipa lagascae Roem. et Schult.

\begin{tabular}{|c|c|}
\hline Chorotype & Life form \\
\hline Med & $\mathrm{T}$ \\
\hline Med & $\mathrm{T}$ \\
\hline Tropical & $\mathrm{He}$ \\
\hline American & Geo \\
\hline Tropical & $\mathrm{T}$ \\
\hline $\mathrm{Sa}-\mathrm{Ar}$ & $\mathrm{He}$ \\
\hline Med & $\mathrm{T}$ \\
\hline Sudanian-African & $\mathrm{He}$ \\
\hline Med & $\mathrm{He}$ \\
\hline Med & $\mathrm{T}$ \\
\hline Med & $\mathrm{T}$ \\
\hline Med & $\mathrm{He}$ \\
\hline Med/ Ir-Tu & $\mathrm{T}$ \\
\hline $\mathrm{Med} / \mathrm{Ir}-\mathrm{Tu}$ & $\mathrm{T}$ \\
\hline Med & $\mathrm{He}$ \\
\hline Med & $\mathrm{T}$ \\
\hline Plurireginalbor-trop & Geo \\
\hline $\mathrm{Med} / \mathrm{Ir}-\mathrm{Tu}$ & $\mathrm{He}$ \\
\hline Med/ Ir-Tu & $\mathrm{He}$ \\
\hline Med & $\mathrm{He}$ \\
\hline Eu-Si/Med/Ir-Tu & $\mathrm{T}$ \\
\hline Eru-Si./Med./Ir-Tu & Geo \\
\hline Med & $\mathrm{T}$ \\
\hline Med & $\mathrm{T}$ \\
\hline Eur-Si/Med & $\mathrm{T} / \mathrm{He}$ \\
\hline $\mathrm{Ir}-\mathrm{Tu}$ & Geo \\
\hline $\mathrm{Eu}-\mathrm{Si} / \mathrm{Med} / \mathrm{Ir}-\mathrm{Tu}$ & $\mathrm{He}$ \\
\hline Med & Geo \\
\hline $\mathrm{Med} / \mathrm{Ir}-\mathrm{Tu}$ & $\mathrm{T}$ \\
\hline $\mathrm{Med} / \mathrm{Ir}-\mathrm{Tu} / \mathrm{Sa}-\mathrm{Ar}$ & $\mathrm{T}$ \\
\hline $\mathrm{Med} / \mathrm{Ir}-\mathrm{Tu}$ & $\mathrm{He}$ \\
\hline Med/ Ir-Tu & $\mathrm{T}$ \\
\hline Tropical & $\mathrm{He}$ \\
\hline $\mathrm{Med} / \mathrm{Ir}-\mathrm{Tu}$ & $\mathrm{He}$ \\
\hline $\mathrm{Med} / \mathrm{Ir}-\mathrm{Tu} / \mathrm{Sa}-\mathrm{Ar}$ & Geo \\
\hline $\mathrm{Ir}-\mathrm{Tu} / \mathrm{Sa}-\mathrm{Ar}$ & $\mathrm{T}$ \\
\hline $\mathrm{Ir}-\mathrm{Tu} / \mathrm{Sa}-\mathrm{Ar}$ & $\mathrm{T}$ \\
\hline $\mathrm{Eu}-\mathrm{Si} / \mathrm{Med} / \mathrm{Ir}-\mathrm{Tu}$ & $\mathrm{T}$ \\
\hline $\mathrm{Med} / \mathrm{Ir}-\mathrm{Tu}$ & $\mathrm{He}$ \\
\hline Plurireginalbor-trop & $\mathrm{T}$ \\
\hline Plurireginalbor-trop & $\mathrm{T}$ \\
\hline $\mathrm{Eu}-\mathrm{Si} / \mathrm{Med} / \mathrm{Ir}-\mathrm{Tu}$ & $\mathrm{T}$ \\
\hline Plurireginalbor-trop & $\mathrm{T}$ \\
\hline Eu-Si/Med & $\mathrm{T}$ \\
\hline $\mathrm{Eu}-\mathrm{Si} / \mathrm{Med} / \mathrm{Ir}-\mathrm{Tu}$ & $\mathrm{T}$ \\
\hline Ir-Tu & $\mathrm{T} / \mathrm{He}$ \\
\hline Subtropical-Tropical & Geo \\
\hline Tropical/Sud & $\mathrm{T}$ \\
\hline $\mathrm{Med} / \mathrm{Ir}-\mathrm{Tu} / \mathrm{Sa}-\mathrm{Ar}$ & $\mathrm{T}$ \\
\hline Med & $\mathrm{T}$ \\
\hline Tropical & Geo \\
\hline Med/Trop & Geo \\
\hline Ir-Tu, ES Eu-Sib, M Med, & $\mathrm{He}$ \\
\hline $\mathrm{Ir}-\mathrm{Tu} / \mathrm{Sa}-\mathrm{Ar}$ & $\mathrm{He}$ \\
\hline $\mathrm{Ir}-\mathrm{Tu} / \mathrm{Sa}-\mathrm{Ar}$ & $\mathrm{T}$ \\
\hline $\mathrm{Med} / \mathrm{Ir}-\mathrm{Tu}$ & $\mathrm{He}$ \\
\hline
\end{tabular}




\section{Species}

Stipa nitens Ball.

Stipa parviflora Desf.

Stipa tenacissima L.

Stipagrostis acutiflora (Trin. et Rupr.) de Winter.

Stipagrostis ciliata (Desf.) de Winter.

Stipagrostis foexiana (Marie et Wilczek) de Winter.

Stipagrostis libyca (H. Scholz) H. Scholz.

Stipagrostis multinerva H. Scholz.

Stipagrostis obtusa (Delile) Nees.

Stipagrostis plumosa (L.) Munro ex T. Anders.

Stipagrostis pungens (Desf.) de Winter.

Stipagrostis rigidifolia $\mathrm{H}$. Scholz.

Stipagrostis scoparia (Trin. Et Rupr.) de Winter.

Stipagrostis shawii (H. Scholz) H. Scholz.

Stipagrostis vulnerans (Trin. Et Rupr.) de Winter.

Stipagrostis zittelii (Aschers.) de Winter.

Tetrapogen villosus Desf.

Trachynia distachya (L.) Link.

Tragus racemosus (L.) All.

Triplachne nitens (Guss.) Link.

Trisetaria glumacea (Boiss) Marie.

Trisetaria linearis Forsk.

Trisetaria macrochaeta (Boiss.) Marie.

Trisetaria vaccariana (Marie et Weiller) Marie.

Triticum aestivum $\mathrm{L}$.

Triticum bicorne Forsk.

Triticum compactum Host.

Triticum durum Desf.

Triticum polonicum $\mathrm{L}$.

Triticum spelta L.

Vulpia bromoides (L.) S. F. Gray.

Vulpia ciliata Dumort.

Vulpia gracilis $\mathrm{H}$. Scholz in

Vulpia inops (Del.) Hackel.

Vulpia ligustica (All.) Link.

Vulpia membranacea (L.) Dumort.

Vulpia myuros (L.) C. C. Gmel.

Vulpiella tenuis (Tineo) Kerguelen.

Zea mays L.

\section{References}

[1] Peterson, P. M. (2013) Poaceae (Gramineae). In: eLS. John Wiley \& Sons, Ltd: Chichester. doi: 10.1002/9780470015902. a0003689. pub2.

[2] Hodkinson, T. R. (2018) Evolution and Taxonomy of the Grasses (Poaceae): A Model, Family for the Study of Species-Rich Groups. Annual Plant Reviews, 1, 1-39. doi: 10.1002/9781119312994. apr0622.

[3] Kellogg, E. A. (2015) Flowering plants. Monocots: Poaceae. In: The Families and Genera of Vascular Plants, vol. 13 (ed. K. Kubitski), 1-416. Cham: Springer International.

[4] Soreng, R. J; Peterson, P. M; Romaschenko, K; Davidse, G; Teisher, J. K; Clark, L. G; Barbera, P; Gillespie, L. J; Zuloaga,

\begin{tabular}{|c|c|}
\hline Chorotype & Life form \\
\hline Med & $\mathrm{He}$ \\
\hline $\mathrm{Ir}-\mathrm{Tu}$ & $\mathrm{He}$ \\
\hline Unclear & Geo \\
\hline Med & $\mathrm{T} / \mathrm{He}$ \\
\hline $\mathrm{Sa}-\mathrm{Ar}$ & $\mathrm{He}$ \\
\hline $\mathrm{Med} / \mathrm{Sud}$ & $\mathrm{He}$ \\
\hline Med & $\mathrm{T}$ \\
\hline $\mathrm{Med} / \mathrm{Ir}-\mathrm{Tu}$ & $\mathrm{He}$ \\
\hline $\mathrm{Sa}-\mathrm{Ar} / \mathrm{Sud}$ & $\mathrm{He}$ \\
\hline $\mathrm{Ir}-\mathrm{Tu} / \mathrm{Sa}-\mathrm{Ar}$ & $\mathrm{He}$ \\
\hline Unclear & Geo \\
\hline $\mathrm{med} / \mathrm{Sud}$ & $\mathrm{He}$ \\
\hline $\mathrm{Sa}-\mathrm{Ar}$ & $\mathrm{He}$ \\
\hline Sudanian & $\mathrm{T}$ \\
\hline Sud & Geo \\
\hline Med & $\mathrm{T} / \mathrm{He}$ \\
\hline Sa-Ar/Sud & $\mathrm{He}$ \\
\hline $\mathrm{Med} / \mathrm{Ir}-\mathrm{Tu}$ & $\mathrm{T}$ \\
\hline Eu-Si/Med & $\mathrm{T}$ \\
\hline Med & $\mathrm{T}$ \\
\hline $\mathrm{Sa}-\mathrm{Ar}$ & $\mathrm{T}$ \\
\hline $\mathrm{Med} / \mathrm{Sa}-\mathrm{Ar}$ & $\mathrm{T}$ \\
\hline $\mathrm{Sa}-\mathrm{Ar}$ & $\mathrm{T}$ \\
\hline Med & $\mathrm{T}$ \\
\hline Exotic, Planted, Escaped from cultivation & $\mathrm{T}$ \\
\hline Med & $\mathrm{T}$ \\
\hline Trop & $\mathrm{T}$ \\
\hline Exotic, Planted, Escaped from cultivation & $\mathrm{T}$ \\
\hline $\mathrm{Med} / \mathrm{Ir}-\mathrm{Tu}$ & $\mathrm{T}$ \\
\hline Eu-Si/Ir-Tu & $\mathrm{T}$ \\
\hline Eu-Si/Med/Trop & $\mathrm{T}$ \\
\hline Eu-Si/Med/Ir-Tu & $\mathrm{T}$ \\
\hline Med & $\mathrm{T}$ \\
\hline Med & $\mathrm{T}$ \\
\hline Med & $\mathrm{T}$ \\
\hline Med & $\mathrm{T}$ \\
\hline Eru-Si./Med./Ir-Tu & $\mathrm{T}$ \\
\hline Med & $\mathrm{T}$ \\
\hline $\operatorname{Cos}$ & $\mathrm{T}$ \\
\hline
\end{tabular}

F. O. (2017) A worldwide phylogenetic classification of the Poaceae (Gramineae) II. An update and comparison of two 2015 classifications. Journal of Systematics and Evolution, 55, 259-290 doi 10.1111/jse.12262.

[5] Saarela, J. M; Burke, S. V; Wysocki, W. P; Barrett, M. D; Clark, L. G; Craine, J. M; Peterson, P. M; Soreng, R. J; Vorontsova, M. S; Duvall, M. R. (2018) A 250 plastome phylogeny of the grass family (Poaceae): topological support under different data partitions. PeerJ 6: e4299; DOI 10.7717/peerj.4299.

[6] Hoque, E; Hossain, A; Rana, S. (2019) Evaluation of Analgesic, Antidiarrheal and Anti-hyperglycemic Activities of Dactyloctenium australe (Poaceae). Bangladesh Pharmaceutical Journal 22 (1): 85-91.

[7] Sagar A., Tajkia J. E. and Sarwar A. K. M. G.(2018) Weed diversity of the family Poaceae in Bangladesh Agricultural University campus and their ethnobotanical uses. Journal of Bangladesh Agricultural University, 16 (3): 372-379. 
[8] Peterson, P. M; Romaschenko, K \& Johnson, G. (2010) A classification of the Chloridoideae (Poaceae) based on multi-gene phylogenetic trees. Molecular Phylogenetics and Evolution, 55, pp. 580-598, ISSN 1095-9513.

[9] Dashora. K \& Gosavi, K. G. C. (2013) Grasses: An Underestimated Medicinal Repository. Journal of Medicinal Plants Studies, 1 (3), 151-157.

[10] Vorontsova, M. S; Clayton, D; Simon, B. K. (2015) Grassroots e-floras in the Poaceae: growing Grass Base and Grass World. PhytoKeys, 48, 73-84. doi: 10.3897/phytokeys.48.7159.

[11] Landi, S; Hausman, J. F; Guerriero, G \& Esposito, S. (2017) Poaceae vs. Abiotic Stress: Focus on Drought and Salt Stress, Recent Insights and Perspectives. Front. Plant Sci., 8, 1214. doi: $10.3389 /$ fpls.2017.01214.

[12] Shen, Q; Fu, L; Dai, F; Jiang, L; Zhang, G; Wu, D. (2016) Multi-omics analysis reveals molecular mechanisms of shoot adaption to salt stress in Tibetan wild barley. BMC Genomics, 17, 889. doi: 10.1186/s12864-016-3242-9.

[13] Arabacı, T \& Yıldız, B. (2004) A floristical study on Poaceae spp., growing naturally in Malatya Province. Turk J Bot., 28, 361-368.

[14] Türe, C; Bingol A. N; Middleton, B. (2004) Characterization of the habitat of Lythrum salicaria L., in Floodplain Forests in Western Turkey - Effects on the Stem height and seed production. Wetlands, 24 (3), 711-716.

[15] El-Mokasabi, F. M. (2017) Studies on the Flora of Libya [Version 1; awaiting peer review]. ContROL 1: 08. doi: 10.28915/control.0008.1.

[16] El-Darier, S. M \& El-Mogaspi, F. M. (2009) Ethnobotany and relative importance of some endemic plant species at El-Jabal El-Akhdar region (Libya). World J. of Agric. Sci. 5 (3), 353-360.

[17] Boulos, L. (1972) our present knowledge on the Flora and Vegetation of Libya. Bibliography. Webbia, 26 (11), 365-400.

[18] Qaiser, M \& El-Gadi, A. A. (1984) Critical analysis of the flora of Libya. Libyan Science Journal, 13, 31-40.

[19] Sharashy, O. S. (2016) New plant records for the Flora of Libya Journal of Sebha University-Pure and Applied Sciences, 15 (2) pp. 105-111.

[20] Sherif, A. S \& Siddiqi, M. A. (1988) Poaceae. In El-Gadi, A., (eds) Flora of Libya, vol. 145. Al Faateh University, Faculty of
Science, the National Herbarium, Department of Botany, Tripoli.

[21] Ardakani, M. R. (2004) Ecology. Tehran University Press, p. 340. Colinvaux P (1993). Ecology. John Wiley and Sons Inc. New York, pp. 648-684.

[22] Eshaghi, R. J; Manthey, M; Mataji, A. (2009) Comparison of plant species diversity with different plant communities in deciduousforests. Int. J. Environ. Sci. Tech., 6 (3), 389-394.

[23] Reich, P. B; Bakken, P; Carlson, D; Frelich, L; Friedman, S. K; Grigal, D. (2001) Influence of logging, fire and forest type on biodiversity and productivity in southern boreal forests. Ecology, 82 (10), pp. 2731-2748.

[24] Ket, M. (2012) Vegetation Description and Data Analysis a Practical Approach ( $2^{\text {nd }}$ edn), John Wiley and Sons Ltd.

[25] Jafri, S. M. H \& Ali, S. L. (1976) Flora of Libya, (1-145). Published by, Al-FaatehUniversity. Faculty of Sciences. Tripoli, Libya: Department of Botany.

[26] Klopper, R. R; Gautier, L; Chatelain, C; Smith, G. F \& Spichiger, R. (2007) Floristics of the angiosperm flora of subSahara African: an analysis of the Africa Plant Checklist and Database. Taxon, 56, 201-208.

[27] Sherif, A. S. (1992) A Revised Key to the Species of the Genus Bromus in Libya Including a New Plant Record. Bulletin, Nat Herb, Tripoli University, Libya. 3, 5-8.

[28] Siddiqi, M. (1992) A New Generic Record for the Libyan Grasses. Bulletin, Nat Herb, TripoliUniversity, Libya 3, 1-4.

[29] Erteeb, F. B \& Sharashi, O. S. (2015) New Records for the Flora of Libya. Libyan Science Journal. 18, 1-14.

[30] Raunkiaer, C. (1934) Life forms of plants and statistical geography, Oxford, $632 \mathrm{P}$.

[31] Govaerts, R; Frodin, D. G \& Radcliffe-Smith, A. (2000) World Checklist and Bibliography of Euphorbiaceae (with Pandanaceae). Volume 1. The Royal Botanic Gardens, Kew.

[32] Kashipazha, A. M; Asri, Y; Moradi, H. M. (2004) Introduction to the flora, lifeformes and Chorology of Bagheshad Region, Iran. Pajouhesh \& Sazandegi, 63, pp 95-103.

[33] Pairanj, J; Ebrahimi, A; Tarnain, F \& Hassanzadeh, M. (2011) Investigation on the geographical distribution and life form of plant species in sub alpine zone Karsanak region, Shahrekord. Journal of Taxonomy and Biosystematics, 3, 1-10. 